HD-THEP-97-12

\title{
Effective Nonlocal Euclidean Gravity
}

\author{
Christof Wetterich $]^{\text {T }}$ \\ Institut für Theoretische Physik \\ Universität Heidelberg \\ Philosophenweg 16, D-69120 Heidelberg
}

\begin{abstract}
A nonlocal form of the effective gravitational action could cure the unboundedness of euclidean gravity with Einstein action. On sub-horizon length scales the modified gravitational field equations seem compatible with all present tests of general relativity and post-Newtonian gravity. They induce a difference in the effective Newton's constant between regions of space with vanishing or nonvanishing curvature scalar (or Ricci tensor). In cosmology they may lead to a value $\Omega<1$ for the critical density after inflation. The simplest model considered here appears to be in conflict with nucleosynthesis, but generalizations consistent with all cosmological observations seem conceivable.
\end{abstract}

\footnotetext{
${ }^{1}$ e-mail C.Wetterich@thphys.uni-heidelberg.de
} 


\section{Stability problem in euclidean gravity}

It is a longstanding observation that the Einstein-Hilbert action for gravity is not bounded in euclidean space. Fundamental problems for the formulation of quantum gravity are connected to this fact. This stability problem in euclidean Einstein gravity can be easily seen if one expands the curvature scalar $R$ around flat space $\mathbb{R}^{d}$ with metric fluctuations $h_{\mu \nu}=g_{\mu \nu}-\delta_{\mu \nu}$. U Up to a total derivative one finds in quadratic order

$$
\begin{aligned}
\sqrt{g} R & =I_{1}-I_{2} \\
I_{1} & =\frac{1}{8}\left(\partial^{\nu} h^{\mu \rho}-\partial^{\rho} h^{\mu \nu}\right)\left(\partial_{\nu} h_{\mu \rho}-\partial_{\rho} h_{\mu \nu}\right) \\
I_{2} & =\frac{1}{4}\left(\partial_{\nu} h^{\rho \nu}-\partial^{\rho} h_{\nu}^{\nu}\right)\left(\partial_{\mu} h_{\rho}^{\mu}-\partial_{\rho} h_{\mu}^{\mu}\right) .
\end{aligned}
$$

One observes that for $d>2$ the euclidean Einstein action $\left(M_{p}=G^{-1 / 2} \approx 10^{19} \mathrm{GeV}\right)$

$$
S_{E}=\frac{M_{p}^{2}}{16 \pi} \int d^{d} x \sqrt{g} R
$$

can become arbitrarily negative for configurations with arbitrarily large $I_{2}$. This becomes obvious by decomposing $h_{\mu \nu}$ into irreducible representations of the Poincaré group

$$
\begin{aligned}
h_{\mu \nu} & =b_{\mu \nu}+\partial_{\mu} a_{\nu}+\partial_{\nu} a_{\mu}+\left(\partial_{\mu} \partial_{\nu}-\frac{1}{d} \delta_{\mu \nu} \partial^{2}\right) \chi+\frac{1}{d} \delta_{\mu \nu} \sigma \\
\partial_{\mu} b^{\mu \nu} & =0, \partial_{\mu} a^{\mu}=0, b_{\mu \nu} \delta^{\mu \nu}=0
\end{aligned}
$$

where

$$
\sqrt{g} R=\frac{1}{4} \partial^{\rho} b^{\mu \nu} \partial_{\rho} b_{\mu \nu}-\frac{(d-1)(d-2)}{4 d^{2}} \partial_{\mu}\left(\sigma-\partial^{2} \chi\right) \partial^{\mu}\left(\sigma-\partial^{2} \chi\right) .
$$

For $d>2$ the Einstein-action becomes negative semi-definite for configurations with vanishing $b_{\mu \nu}$. A general gauge fixing term

$$
S_{g f}=\frac{M_{p}^{2}}{64 \pi} \int d^{d} x\left(\eta_{1} \partial_{\nu} h^{\rho \nu}-\eta_{2} \partial^{\rho} h_{\nu}^{\nu}\right)\left(\eta_{1} \partial_{\mu} h_{\rho}^{\mu}-\eta_{2} \partial_{\rho} h_{\mu}^{\mu}\right)
$$

can move the unboundedness to other irreducible representations but not cure the disease.[ The unboundedness of $S_{E}+S_{g f}$ can also not be stabilized by higher powers of $h$ since we can always consider small but arbitrarily strongly varying $h$. As a

\footnotetext{
${ }^{2}$ Our conventions are [1] $R=R_{\mu \nu} g^{\mu \nu}=R_{\mu}^{\mu}, R_{\mu \nu}=R_{\mu \lambda \nu}{ }^{\lambda}, R_{\mu \nu \rho}^{\lambda}=\partial_{\mu} \Gamma_{\nu \rho}^{\lambda}-\partial_{\nu} \Gamma_{\mu \rho}{ }^{\lambda}+$ $\Gamma_{\mu \tau}{ }^{\lambda} \Gamma_{\nu \rho}{ }^{\tau}-\Gamma_{\nu \tau}{ }^{\lambda} \Gamma_{\mu \rho}{ }^{\tau}, g=\operatorname{det}\left(g_{\mu \nu}\right)$. As compared to earlier work of the same author, we use here an opposite sign of the definition of $R_{\mu \nu \rho}{ }^{\lambda}$. For a continuation to Minkowski space the signature will be $(-+++)$, also opposite to earlier work of the author.

${ }^{3}$ For this argument it is important to observe that $\eta_{1}=\eta_{2}$ is not an allowed gauge fixing. Otherwise $\eta_{1}$ and $\eta_{2}$ are arbitrary constants.
} 
result, no well defined partition function $Z=\int D g_{\mu \nu} e^{-\left(S_{E}+S_{g f}+S_{g h}\right)}$ can be defined with the Einstein-action for standard measures $D g_{\mu \nu}$ respecting general covariance.f

By itself, the unboundedness of Einstein's action poses no severe problem for the quantization of gravity by a functional integral. There are many possible extensions of this action which only influence the short distance behaviour and make the action bounded. One of the simplest is the addition of a term $\int d^{d} x \sqrt{g} R^{2}$ with a positive coefficient. The partition function in presence of sources is then well defined (in presence of a suitable ultraviolet regularization) and one may compute the effective action $\Gamma$ as the generating functional of the 1PI Green functions. For any classical action which is bounded from below, however, the effective action should also be bounded from below. The instability problem reappears now for any effective action for which only the Einstein term (2) (plus gauge fixing) is relevant for the long distance physics. The absolute minimum of such an effective action cannot correspond to flat space. We know from eq. (4) that there are necessarily configurations which have a lower effective action as compared to $g_{\mu \nu}=\delta_{\mu \nu}$. The absolute minimum of the (bounded) effective action should therefore correspond to a nonvanishing curvature tensor. Despite the well known classical stability for small fluctuations around Minkowski space (positive energy theorem) there remains the open question why the observed ground state of gravity (flat space) is not given by the minimum of the euclidean effective action. Interpreting as usual $\exp (-\Gamma)$ as a probability distribution the flat space configuration $g_{\mu \nu}=\delta_{\mu \nu}$ would be exponentially suppressed as compared to the absolute minimum of $\Gamma$. With a normalization $\Gamma\left[g_{\mu \nu}=\delta_{\mu \nu}\right]=0$ the latter would necessarily occur for negative $\Gamma$ and have non-zero curvature.

In this letter we adopt the attitude that the effective euclidean action for gravity should have its absolute minimum for flat space (in absence of matter). This means that the long-distance behaviour of the effective action needs an extension beyond Einstein gravity. We will investigate a nonlocal effective action for gravity and discuss possible observational consequences. In view of the fundamental character of the euclidean stability problem it seems justified to ask which extensions of Einstein gravity are allowed phenomenologically without presenting a quantum field theoretical justification of the assumed nonlocalities.

\section{Nonlocal gravity}

How could an effective action for gravity at long distances look like? We will require here general covariance and positivity for the euclidean action, with $\Gamma=0$ for flat

\footnotetext{
${ }^{4}$ General covariance forbids an arbitrary change of sign of the second term on the r.h.s. of eq. (4). This would result for an analytic continuation from Minkowski space where the conformal mode would be rotated with the opposite sign as compared to the transversal modes [2]. The precise form of the ghost action $S_{g h}$ is unimportant for our problem and we have not written explicitly the necessary functional integration over ghost fields.

5 This holds after subtraction of a suitable constant.
} 
space.f By long distances we mean momentum scales much smaller than $M_{p}$. It is rather obvious that no local effective action can fulfil both conditions simultaneously. Adding a cosmological constant term only worsens the stability problem. The Einstein-Hilbert-term and the cosmological constant are the only invariants involving up to two derivatives. Higher derivative terms $\sim R^{2}, R_{\mu \nu} R^{\mu \nu}$ or $R_{\mu \nu \lambda \sigma} R^{\mu \nu \lambda \sigma}$ are ineffective at long distances. They do not remedy the stability problem either. This generalizes to any finite number of derivatives.

One way out of the dilemma seems a nonlocal form of the effective action. Since there are always massless graviton fluctuations around flat space there is a priori no argument why the effective action must be local. Without a consistent nonperturbative formulation of euclidean gravity with an Einstein action we can also not rely on the results of a one-loop calculation of the effective action which is based on a classical Einstein action [3]. In this letter we explore the possibility of a nonlocal effective action which extends Einstein's gravity in a simple way. We are interested in the general consequences of such an extension, without having at the moment indications that quantum gravity really produces the nonlocal features discussed here. Our model is chosen for simplicity, and we have no arguments which favour it as compared to more elaborate nonlocal extensions which we will briefly discuss at the end. For definiteness we consider

$$
\begin{aligned}
\Gamma & =\frac{M_{p}^{2}}{16 \pi} \int d^{d} x\left\{\sqrt{g} R+\frac{1}{2} \tau^{2} \mathcal{L}_{n l}\right\} \\
\mathcal{L}_{n l} & =\sqrt{g} R \mathcal{D}^{-1} R=-\sqrt{g} R\left(\varepsilon R+D^{2}\right)^{-1} R
\end{aligned}
$$

with $D^{2}$ the covariant Laplacian] $D^{2} R=R ;{ }_{\mu}{ }_{\mu}$. We remind here that the inverse of differential operators has to be handled with some care and $\mathcal{D}=-\varepsilon R-D^{2}$ should only be considered as an appropriate limit for a properly regularized operator $\mathcal{D}$. For a given metric $g_{\mu \nu}$ the meaning of $\mathcal{D}^{-1} R$ is specified in a basis of eigenvectors of $\mathcal{D}$

$$
R(x)=\sum_{n} a_{n} R_{n}(x), \mathcal{D} R_{n}(x)=\lambda_{n} R_{n}(x), \mathcal{D}^{-1} R(x)=\sum_{n} a_{n} \lambda_{n}^{-1} R_{n}(x) .
$$

A regularized operator should have the property $\mathcal{D} R=0 \Rightarrow R=0$ such that all $\lambda_{n}$ are different from zero and $R=0 \Rightarrow \mathcal{D}^{-1} R=0$. Translating $\mathcal{D}=-\varepsilon R-D^{2}$ to a Fourier basis one finds for $p^{2} \neq 0$ and $h_{\mu \nu} \rightarrow 0$ that in linear order in $h_{\mu \nu}$ the term $\sim \varepsilon R$ is ineffective

$$
\left(-\left(D^{2}+\varepsilon R\right)^{-1} R\right)(p)=\left(-\left(D^{2}\right)^{-1} R\right)(p)=-\frac{p^{\mu} p^{\nu}}{p^{2}}\left(h_{\mu \nu}(p)-h_{\rho}^{\rho}(p) \delta_{\mu \nu}\right)=\frac{R(p)}{p^{2}} .
$$

The term $\sim \varepsilon$ plays, however, a role in the limit where $D^{2} R \rightarrow 0$ while $R \neq 0$, as for the example of a sphere. In this limit $\left(\varepsilon R+D^{2}\right)^{-1} R$ approaches the constant $1 / \varepsilon$

\footnotetext{
${ }^{6}$ We do not include in our discussion the generalized gauge fixing term which is also present in the effective action and constrained by Ward identitites.

${ }^{7}$ Here $D_{\mu} S_{\nu \rho \ldots}=S_{\nu \rho \ldots ; \mu}$ denotes a covariant derivative.
} 
and this remains so if the radius of the sphere goes to infinity. Obviously, the two limits are distinguished by the relative size of $D^{2} R$ as compared to $R^{2}$. We note that the operator $\mathcal{D}^{-1}$ still needs to be regularized for metrics for which $D^{2} R=-\varepsilon R^{2}$. For our purposes the regularization

$$
\mathcal{D}=-\varepsilon R-D^{2}+\frac{\gamma}{M_{p}^{2}} R_{\mu \nu \sigma \lambda} R^{\mu \nu \sigma \lambda}
$$

will be sufficienty, and we omit the regulator term $\sim \gamma$ unless explicitly needed.

Assuming $\tau^{2}>0$ the new nonlocal piece is positive semi-definite for small fluctuations. In terms of irreducible representations one finds now in quadratic order in $h_{\mu \nu}$

$$
\begin{aligned}
\sqrt{g} R+ & \frac{1}{2} \tau^{2} \mathcal{L}_{n l}=\frac{1}{4} \partial^{\rho} b^{\mu \nu} \partial_{\rho} b_{\mu \nu} \\
& +\left\{\frac{1}{2} \tau^{2}\left(1-\frac{1}{d}\right)^{2}-\frac{(d-1)(d-2)}{4 d^{2}}\right\} \partial^{\mu}\left(\sigma-\partial^{2} \chi\right) \partial_{\mu}\left(\sigma-\partial^{2} \chi\right) .
\end{aligned}
$$

Provided

$$
\tau^{2} \geq \frac{d-2}{2(d-1)}
$$

this expression has indeed its absolute minimum for flat space! It is therefore a candidate for an effective action consistent with our conditions. We note that expressed in terms of irreducible representations $\mathcal{L}_{n l}$ is actually a local expression which takes the form of an additional positive kinetic term for the scalar $\sigma-\partial^{2} \chi$. In consequence, this scalar ceases to be a "tachyon" (with negative sign of the euclidean kinetic term). In other words, $\Gamma$ contains in quadratic order only standard kinetic terms for a traceless transversal symmetric second rank tensor $b_{\mu \nu}$ and a scalar $\sigma-\partial^{2} \chi$. In this language it is particularly easy to see that the limit of flat space $\left(b_{\mu \nu} \rightarrow 0, \sigma \rightarrow 0, \chi \rightarrow 0\right)$ is well behaved despite the operator $\left(-\varepsilon R-D^{2}\right)^{-1}$ in (6).

The linearized gravitational equations (Newtonian limit) can be obtained if we add to the effective action a source term $\Gamma_{T}=\int d^{d} x \mathcal{L}_{T}$ for the conserved linearized energy momentum tensor

$$
\begin{aligned}
& T_{\mu \nu}=V_{\mu \nu}+\left(\partial_{\mu} \partial_{\nu}-\partial^{2} \delta_{\mu \nu}\right) W, T_{\mu}^{\mu}=-(d-1) \partial^{2} W \\
& \partial^{\nu} V_{\mu \nu}=0, \delta^{\mu \nu} V_{\mu \nu}=0
\end{aligned}
$$

With $T^{\mu \nu}=-2 g^{-1 / 2} \delta \Gamma_{T} / \delta g_{\mu \nu}$ one has in the lowest order (upon partial integration)

$$
\mathcal{L}_{T}=-\frac{1}{2} h_{\mu \nu} T^{\mu \nu}=-\frac{1}{2}\left\{b_{\mu \nu} V^{\mu \nu}-\left(1-\frac{1}{d}\right)\left(\sigma-\partial^{2} \chi\right) \partial^{2} W\right\}
$$

\footnotetext{
${ }^{8}$ More general regularizations could easily be written down and $\mathcal{D}$ could be turned into a strictly positive operator. We note that $\mathcal{L}_{n l}$ is uniquely defined also for a flat metric or constant $h_{\mu \nu}$ where it vanishes.
} 
and therefore the field equations read for $d=4$

$$
\begin{aligned}
& \partial^{2} b^{\mu \nu}=-\frac{16 \pi}{M_{p}^{2}} V^{\mu \nu}, \partial^{2} \tilde{\sigma}=\frac{16 \pi}{M_{p}^{2}} \frac{2}{3 \tau^{2}-1} \partial^{2} W \\
& \tilde{\sigma}=\sigma-\partial^{2} \chi
\end{aligned}
$$

Only the field equation for $\tilde{\sigma}$ in presence of matter $\left(T_{\mu}^{\mu} \neq 0\right)$ is influenced by the nonlocal term $\sim \tau$. Continuation to Minkowski space changes the definition of the fields (replacing $\delta_{\mu \nu}$ by $\eta_{\mu \nu}=\operatorname{diag}(-1,+1,+1,+1$ ) in eqs. (3) (12)), but does not affect the field equations (14). The field equation (14) can be cast in the form of the usual linearized Einstein equation if we absorb the contribution from $\mathcal{L}_{n l}$ into a new gravitational contribution to the "total" energy momentum $\hat{T}^{\mu \nu}=T^{\mu \nu}+T_{n l}^{\mu \nu}$, where $V_{n l}^{\mu \nu}=0$ and $W_{n l}$ can be inferred from $\partial^{2} \tilde{\sigma}=-\frac{32 \pi}{M_{p}^{2}}\left(\partial^{2} W+\partial^{2} W_{n l}\right)$ as

$$
\partial^{2} W_{n l}=-\frac{3 \tau^{2}}{3 \tau^{2}-1} \partial^{2} W
$$

In particular, one finds for a static configuration

$$
\hat{T}_{00}=T_{00}+\Delta W_{n l}=T_{00}+\frac{\tau^{2}}{3 \tau^{2}-1} T_{\mu}^{\mu}
$$

and, for $\sum_{i} T_{i i}=0$,

$$
\hat{T}_{00}=\frac{2 \tau^{2}-1}{3 \tau^{2}-1} T_{00}
$$

Up to a rescaling of the effective Newton's constant

$$
G_{N, e f f}=\frac{2 \tau^{2}-1}{3 \tau^{2}-1} \frac{1}{M_{p}^{2}}=\frac{1}{\left(M_{p, e f f}^{(0)}\right)^{2}}
$$

this is standard Newtonian gravity provided $G_{N, \text { eff }}$ is positive. In our context we will impose the condition

$$
\tau^{2}>\frac{1}{2}
$$

Observation of nonlocal gravity at the linearized level requires a measurement of the additional coupling to $T_{i}^{i}$ which appears in $\hat{T}_{00}$ (16) for nonvanishing pressure. At present we are not aware of such a measurement.

Gravitational radiation in empty space or the propagation of gravitons is not affected by nonlocal gravity. Gravitons are described by $b_{\mu \nu}$, and we observe that the source term for gravitons obtains no nonlocal correction in linearized order (eq. (31)). For $\tau \neq 0$ this implies that the value of $G_{N}$ as measured by the emission of gravitational waves should differ from the static value of the effective Newton's constant (18). This is, however, not tested today. Beyond linearized gravity we have to solve the gravitational field equations for nonlocal gravity. 


\section{Gravitational field equations}

The gravitational field equations obtain from the functional derivative of the effective action $\Gamma$ with respect to the metric. For their derivation it is useful to write $\Gamma$ in an equivalent local form by introducing a scalar field $\varphi$

$$
\begin{aligned}
\varphi & =-\tau \mathcal{D}^{-1} R \\
\Gamma & =\frac{M_{p}^{2}}{16 \pi} \int d^{d} x \sqrt{g}\left\{-\frac{1}{2} \varphi \mathcal{D} \varphi+(1-\tau \varphi) R\right\}
\end{aligned}
$$

Inserting the field equation for $\varphi, \mathcal{D} \varphi=-\tau R$, into $\Gamma$ one recovers the original effective action (6). Solutions of the field equations from (20) or (6) are therefore equivalent. f For $\mathcal{D}=-\epsilon R-D^{2}$ the field equation for $\varphi$ and the effective action are given by

$$
\begin{aligned}
& D^{2} \varphi=(\tau-\varepsilon \varphi) R \\
& \Gamma=\frac{M_{p}^{2}}{16 \pi} \int d^{d} x \sqrt{g}\left\{-\frac{1}{2} D^{\mu} \varphi D_{\mu} \varphi+\left(1-\tau \varphi+\frac{1}{2} \varepsilon \varphi^{2}\right) R\right\} .
\end{aligned}
$$

We emphasize that the form (21) should only be considered as an auxiliary action for on-shell $\varphi$ obeying (21). For off-shell fluctuations of $\varphi$ the wrong sign kinetic term would produce new instabilities. On the other hand, for on-shell $\varphi$ one has, of course, a contribution which is positive definite for positive eigenvalues of $\left(-\varepsilon R-D^{2}\right)$

$$
\frac{1}{2} \tau^{2} \mathcal{L}_{n l}=\frac{1}{2} \sqrt{g}\left(D^{\mu} \varphi D_{\mu} \varphi-\varepsilon R \varphi^{2}\right) .
$$

The gravitational field equations can now be found by variation of (21) with respect to the metric

$$
\begin{aligned}
R_{\mu \nu}-\frac{1}{2} R g_{\mu \nu}= & -\frac{1}{1-\tau \varphi+\frac{1}{2} \varepsilon \varphi^{2}}\left\{\left(-\frac{1}{2}+\varepsilon\right) D_{\mu} \varphi D_{\nu} \varphi+\left(\frac{1}{4}-\varepsilon\right) D^{\rho} \varphi D_{\rho} \varphi g_{\mu \nu}\right. \\
& \left.+(\varepsilon \varphi-\tau)\left(D_{\nu} D_{\mu} \varphi-D^{2} \varphi g_{\mu \nu}\right)+\frac{8 \pi}{M_{p}^{2}} T_{\mu \nu}\right\} .
\end{aligned}
$$

Here we have added the energy momentum tensor $T_{\mu \nu}$ for matter or radiation. With (21) this implies for the curvature scalar for $d=4$

$$
\begin{aligned}
R= & \frac{8 \pi}{M_{p}^{2}}\left[1-3 \tau^{2}-(1-6 \varepsilon)\left(\tau-\frac{1}{2} \varepsilon \varphi\right) \varphi\right]^{-1} \\
& \left(T_{\mu}^{\mu}+\frac{M_{p}^{2}}{16 \pi}(1-6 \varepsilon) D_{\mu} \varphi D^{\mu} \varphi\right)
\end{aligned}
$$

\footnotetext{
${ }^{9}$ Note that the general local solution of $\mathcal{D} \varphi=-\tau R$ has free integration constants which must be fixed by appropriate boundary conditions according to (7). The integration constants are related to a function $\psi$ obeying $\mathcal{D} \psi=0$ that can be added to the local solution for $\varphi$. In particular, the function $\psi$ must be chosen such that $\varphi$ vanishes for $\tau=0$ or for a uniformly vanishing curvature scalar. Also $\varphi$ should have no singularity if $R$ is nonsingular. More formally, $\varphi$ must belong to a space of functions for which $\mathcal{D} \varphi=0 \Rightarrow \varphi=0$.
} 
and therefore

$$
D^{2} \varphi=\frac{8 \pi}{M_{p}^{2}} \quad \frac{\varepsilon \varphi-\tau}{3 \tau^{2}-1+(1-6 \varepsilon)\left(\tau-\frac{1}{2} \varepsilon \varphi\right) \varphi}\left(T_{\mu}^{\mu}+\frac{M_{p}^{2}}{16 \pi}(1-6 \varepsilon) D^{\mu} \varphi D_{\mu} \varphi\right) .
$$

It is easy to check that eq. (23) with $D^{2} \varphi=(\tau-\varepsilon \varphi) R$ implies $T_{; \nu}^{\mu \nu}=0$. The field equations are the same for Euclidean or Minkowski signature of the metric.

In this language it is obvious that the nonlocal term $\mathcal{L}_{n l}$ remains compatible with all solutions of Einstein's field equation in empty space. This means that an arbitrary solution of the Einstein-equation with vanishing energy momentum tensor, $R_{\mu \nu}-\frac{1}{2} R g_{\mu \nu}=0$, remains a solution of nonlocal gravity. In fact, for $R=0$ the field equation (23) has always the trivial solution $\varphi=0$. The additional terms $\sim \tau$ in the gravitational field equation (23) vanish for $\varphi=0$ and do therefore not affect the solutions of the Einstein-equation. In particular, there are no corrections to post Newtonian gravity in contrast to Brans-Dicke-theory or general scalar-tensor theories. For example, far away from an extended massive object one has the standard Schwarzschild metric.

There is, however, one notable difference from Einstein gravity, namely that the total energy of the object, which appears in the integration constant $M$ of the Schwarzschild solution, gets a modified contribution from the gravitational energy. It is given by

$$
M=\int_{V} d^{3} x\left(T_{00}+t_{o o}\right)
$$

with $t_{00}$ defined implicitly by linearizing eq. (23) in an asymptotically Minkowskian coordinate system [1]

$$
R_{00}^{(1)}-\frac{1}{2} \eta_{00} R^{(1)}=-\frac{8 \pi}{M_{p}^{2}}\left(T_{00}+t_{00}\right)
$$

The nonlocal contribution to $t_{00}$ beyond Einstein gravity is found from eq. (23) for static $\varphi$

$$
\begin{aligned}
& t_{n l, 00}=\frac{\varphi\left(\tau-\frac{1}{2} \epsilon \varphi\right)}{1-\tau \varphi+\frac{1}{2} \epsilon \varphi^{2}} T_{00}- \\
& -\frac{(\tau-\epsilon \varphi)^{2} T_{\mu}^{\mu}-\frac{M_{p}^{2}}{32 \pi}\left[\tau^{2}-1+4 \epsilon+(1-6 \epsilon)\left(\tau-\frac{1}{2} \epsilon \varphi\right) \varphi\right] \partial^{i} \varphi \partial_{i} \varphi}{\left(1-\tau \varphi+\frac{1}{2} \epsilon \varphi^{2}\right)\left[3 \tau^{2}-1+(1-6 \epsilon)\left(\tau-\frac{1}{2} \epsilon \varphi\right) \varphi\right]} g_{00}
\end{aligned}
$$

One recovers the Newtonian limit (17) for $\varphi \rightarrow 0$ and $g_{00} \rightarrow-1$. We observe that eq. (25) also admits the constant solution $\varphi=\tau / \epsilon$ for which one finds $t_{n l, 00}=-\frac{\tau^{2}}{\tau^{2}-2 \epsilon} T_{00}$. In this regime the nonlocal gravitational energy has a tendency to cancel the normal part $T_{00}$ for $\epsilon<0$ and to enhance it for $\epsilon>\frac{1}{2} \tau^{2}$.

We conclude that the difference between nonlocal gravity and Einstein gravity manifests itself only in different effective couplings of the metric to the energymomentum tensor - in empty space both are identical. For a computation of the 
modified coupling we may distinguish two regimes, for $\epsilon R$ or $D^{2}$ dominating the operator $\mathcal{D}$. A rough criterion for the domination of the $\epsilon R$ term (i.e. $\varphi \approx \tau / \epsilon$ ) is $\rho / M_{p}^{2} \gg L^{-2}$ with $\rho$ the mass density and $L^{-1}$ a typical gradient of the mass distribution. For $m \approx \rho L^{3}$ the total mass of the system this translates into a bound for the density $\rho / M_{p}^{4} \gg M_{p}^{2} / m^{2}$. For $m$ of the order of a solar mass the critical density $\rho_{c} \approx(1 \mathrm{GeV})^{4}$ is roughly of the order of nuclear density. Without going into more details, it seems plausible that ordinary stars can well be described by Newtonian gravity with the effective coupling (18). Modifications of the gravitational coupling to neutron stars are possible and we expect very sizable changes for the interior of black holes. For $\epsilon<\frac{1}{2} \tau^{2}$ the effective gravitational coupling to mass in the interior of a black hole is weaker than in Einstein gravity and the attractive force therefore reduced. It would be interesting to investigate possible effects on the singularity in the center of a black hole.

\section{Cosmology}

Since the present version of nonlocal gravity is compatible with those tests of general relativity which are based on solutions of Einstein equations in empty space, it can only be detected by anomalies in the gravitational coupling to matter. A promising test is cosmology. On cosmological scales space is not empty and the curvature scalar does not always vanish. For $R \neq 0$ there is a source for $\varphi$ (eq. (21)) and nonlocal gravity leads to modifications of the cosmological equations. For a general homogenous and isotropic metric with scale factor $a(t)$ one has (for Minkowski signature $(-+++)$, zero spatial curvature $k=0, d=4$ and $H=\dot{a} / a$ )

$$
\begin{aligned}
R_{00} & =3 \ddot{a} / a=3\left(H^{2}+\dot{H}\right) \\
R_{i j} & =-\left(\ddot{a} / a+2 \dot{a}^{2} / a^{2}\right) g_{i j}=-\left(3 H^{2}+\dot{H}\right) g_{i j} \\
R & =-6\left(\dot{a}^{2} / a^{2}+\ddot{a} / a\right)=-\left(12 H^{2}+6 \dot{H}\right) \\
\varphi_{; 00} & =\ddot{\varphi}, \varphi_{; i j}=-H \dot{\varphi} g_{i j} .
\end{aligned}
$$

This yields the field equations

$$
\begin{gathered}
D^{2} \varphi=-(\ddot{\varphi}+3 H \dot{\varphi})=-a^{-3} \frac{d}{d t}\left(\dot{\varphi} a^{3}\right) \\
=-(\varepsilon \varphi-\tau) R=6(\varepsilon \varphi-\tau)\left(2 H^{2}+\dot{H}\right) \\
H^{2}=\frac{1}{\left(1-\tau \varphi+\frac{1}{2} \varepsilon \varphi^{2}\right)}\left\{\frac{8 \pi}{3 M_{p}^{2}} \rho-\frac{1}{12} \dot{\varphi}^{2}+(\tau-\varepsilon \varphi) H \dot{\varphi}\right\} \\
\dot{\rho}+n H \rho=0
\end{gathered}
$$

with $n=3$ or 4 for a matter or radiation dominated universe. Equation (25) now reads

$$
\ddot{\varphi}+3 H \dot{\varphi}=\frac{1}{2} \quad \frac{(\varepsilon \varphi-\tau)\left(T+(1-6 \varepsilon) \dot{\varphi}^{2}\right)}{3 \tau^{2}-1+(1-6 \varepsilon)\left(\tau-\frac{1}{2} \varepsilon \varphi\right) \varphi}
$$


with

$$
T=-\frac{16 \pi}{M_{p}^{2}} T_{\mu}^{\mu}=\frac{16 \pi}{M_{p}^{2}}(4-n) \rho,
$$

and we remind that $\tau^{2}>\frac{1}{2}(\sqrt{19})$.

For the radiation dominated universe with $n=4$ and $T=0$ we recover the standard Friedman solution

$$
H=\frac{1}{2} t^{-1}, \rho=\rho_{0} t^{-2}, \varphi=0, \quad \rho_{0}=\frac{3 M_{p}^{2}}{32 \pi} .
$$

This is easily understood since for this solution the curvature scalar vanishes. There are therefore no corrections from the nonlocal term in the effective action. As an important consequence all cosmological predictions from the radiation dominated epoch are only modified by the difference between $M_{p}$ and $M_{p}^{(0)}$ eff. For nucleosynthesis this implies that for a given temperature $H^{2}$ is larger than its value in Einstein gravity by a factor $\left(3 \tau^{2}-1\right) /\left(2 \tau^{2}-1\right)>\frac{3}{2}$. Such a big change in the gravitational clock seems not compatible with the successful cosmological explanation of nucleosynthesis and the solution (35) with $\varphi=0$ is excluded! We remark, however, that this is not the only solution of the system of differential equations (31)-(24). For example, any constant $\varphi$ would correspond to a solution, but with a modified critical density $\rho_{0}$ or, equivalently, a modified effective $M_{p}$. (These are actually stable asymptotic solutions for initial conditions with $\dot{\varphi} \neq 0$.) Nevertheless, only $\varphi=0$ is compatible with the regularization of $\mathcal{D}$ if the curvature scalar vanishes identically. Below we will come back to possible effects of a nonvanishing $R$ during the radiation-dominated period.

The situation changes for the epoch before the universe was radiation dominated (inflation), and, most striking, for the matter dominated period. The curvature scalar does not vanish in these cases and $\varphi=0$ remains not a solution anymore. We observe that the system (30) - (23) exhibits a solution with static $\varphi$, namely

$$
\varphi=\frac{\tau}{\epsilon}
$$

This simply results in a multiplication of the effective $M_{p}^{2}$ by a factor $1-\frac{\tau^{2}}{2 \epsilon}$

$$
\left(M_{p, e f f}^{(c)}\right)^{2}=M_{p}^{2}\left(1-\frac{\tau^{2}}{2 \epsilon}\right)
$$

For $\epsilon>\frac{1}{2} \tau^{2}$ or $\epsilon<0$ this implies for the matter dominated epoch a solution

$$
H=\frac{2}{3} t^{-1}, \quad \rho=\frac{16}{9}\left(1-\frac{\tau^{2}}{2 \epsilon}\right) \rho_{0} t^{-2}
$$

As in standard matter dominated cosmology one has $D^{2} R=2 t^{-2} R$. The solution $\varphi / \tau=\left(\varepsilon R+D^{2}\right)^{-1} R=1 / \varepsilon$ seems somewhat surprising since it is the same as for a covariantly constant curvature scalar. It is, however, the general result whenever 
the operator $\left(R+\frac{1}{\varepsilon} D^{2}\right)^{-1}=(R+x)^{-1}$ can be expanded in the differential operator $x(x \cdot 1=0)$, i.e. $(R+x)^{-1} R=\left(1-\frac{1}{R} x+\frac{1}{R} x \frac{1}{R} x-\ldots\right) 1=1$. Of course, this holds only approximately in the limit where the regulator term $\sim \gamma$ in $(9)$ is neglected. $\square$ The solution (36), (38) is an attractor in the space of general solutions of eqs. (30-32) for $t \rightarrow \infty$. For a smooth transition between the radiation and matter-dominated epoch the particular solution with the asymptomatic behaviour (19), (23) should be selected such that it connects continuously with the value of $\varphi=0$ before the transition. Comparing eq. (38) with the critical density in Einstein gravity $\rho_{c}^{(E)}$, we find that the value of $\Omega$ for a zero curvature $(k=0)$ universe as predicted by inflation is

$$
\Omega=\frac{\rho_{c}}{\rho_{c}^{(E)}}=\frac{2 \tau^{2}-1}{3 \tau^{2}-1}\left(1-\frac{\tau^{2}}{2 \epsilon}\right)
$$

For positive $\epsilon>\frac{1}{2} \tau^{2}$ one finds $0<\Omega<\frac{2}{3}$ and also for negative $\epsilon<\frac{1}{2}-\tau^{2}$ the effective critical density turns out to be smaller than one. The value preferred by present observation $\Omega \approx 0.3-0.5$ seems well compatible with nonlocal gravity.

In conclusion, the only discrepancy with observation for the simple model of nonlocal gravity (6) appears to be nucleosynthesis. The extent to which the effective Planck mass differs between nuclosynthesis and today depends, however, crucially on the precise form of the regularized operator $\mathcal{D}$ : Due to the dilatation anomaly (running couplings) and presence of mass corrections in the equation of state the curvture scalar does actually not vanish exactly during the radiation-dominated epoch. Therefore the effective Newton's constant could also deviate from $1 / M_{p}^{2}$ for this period. Furthermore, generalized nonlocal terms of the type

$$
\mathcal{L}_{n l}=\sqrt{g} R^{\mu \nu}\left(\tilde{\mathcal{D}}^{-1}\right)_{\mu \nu \sigma \lambda} R^{\sigma \lambda}
$$

with $\tilde{\mathcal{D}}$ an appropriately regularized differential operator could also cure the unboundedness of the euclidean effective action. (Eq. (6) corresponds to a special case of $\tilde{\mathcal{D}}$.) Again, there will be no changes in the gravitational solutions for empty space since $R_{\mu \nu}=0$. On the other hand $R_{\mu \nu}$ differs from zero during the radiationdominated period in early cosmology, thus leading to an effective value of the Planck mass different from $M_{p}$ during nucleosynthesis. Without a detailed investigation of possible forms of $\tilde{\mathcal{D}}$ in eq. (40) it seems difficult to exclude nonlocal gravity on the basis of nucleosynthesis. For the moment there seem to be too many free parameters such that the effective values of the Planck mass during the radiation and matter dominated periods remain essentially undetermined. Nucleosynthesis can then be interpreted as a "measurement" of the difference in $M_{p}^{e f f}$ between the radiationdominated cosmology and the Newtonean value (18). Within certain assumptions

\footnotetext{
${ }^{10}$ For $\gamma>0$ one has with $\mathcal{D}=y-D^{2}$ the expansion $\left(y-D^{2}\right)^{-1} R=$ $-\frac{1}{\varepsilon}\left\{1-\frac{\gamma}{M_{p}^{2}}\left(1+y^{-1} D^{2}+\left(y^{-1} D^{2}\right)^{2}+\left(y^{-1} D^{2}\right)^{3}+\ldots\right) y^{-1} R_{\mu \nu \sigma \lambda} R^{\mu \nu \sigma \lambda}\right\}$. For $H=\eta t^{-1}$ and $|\varepsilon R| \gg\left(\gamma / M_{p}^{2}\right) R_{\mu \nu \sigma \lambda} R^{\mu \nu \sigma \lambda}$ this yields $\mathcal{D}^{-1} R \approx-\frac{1}{\varepsilon}-\frac{\gamma}{\varepsilon^{2} M_{p}^{2}}\left(1+\frac{1-\eta}{\varepsilon \eta(2 \eta-1)}\right)^{-1} R_{\mu \nu \sigma \lambda} R^{\mu \nu \sigma \lambda} / R$ (if $\left.|\varepsilon|>\frac{1-\eta}{\eta(2 \eta-1)}\right)$, whereas in the opposite limit $R / R_{\mu \nu \sigma \lambda} R^{\mu \nu \sigma \lambda} \rightarrow 0$ one finds $\mathcal{D}^{-1} R \rightarrow 0$.
} 
a determination of $\Omega$ can play a similar role for the "measurement" of $M_{p}^{e f f}$ during matter dominated cosmology. It is striking that it seems not possible to establish the validity of the Einstein equations for long-distance gravity using only the principle of general covariance and phenomenological considerations based on the presently available tests of general relativity. On the other hand, some models of nonlocal gravity, especially involving generalizations of $\mathcal{L}_{n l}$ beyond (40), may lead to more drastic observable modifications of phenomenology.

Of course, the most crucial question remains if quantum gravity really leads to an effective action of the type (40). A one-loop computation in pure Einstein gravity gives no indication in this direction [3] since the nonlocalities appear here only logarithmic. The compatibility of our simple model of nonlocal gravity with observation should motivate further non-perturbative studies of long-distance quantum gravity, perhaps based on exact flow equations [ [1] and not assuming necessarily a "classical action" of the pure Einstein type. We hope that this work motivates observational and experimental efforts to look for a possible curvature dependence of the effective Newton's constant.

\section{References}

[1] S. Weinberg, "Gravitation and Cosmology", John Wiley, 1972

[2] S. Hawking, "Relativity, groups and topology II" (Les Houches 1983), North Holland

[3] G. 't Hooft, M. Veltman, Ann. Inst. Henri Poincaré 20 (1974) 69;

B. DeWitt, "Relativity, groups and topology II" (Les Houches 1983), North Holland;

A. O. Barvinski, Y. V. Gusev, G. A. Vilkovisky, and V. V. Zhytnikov, Nucl. Phys. B439 (1995) 561;

A. G. Mirzabekian, G. A. Vilkovisky, and V. V. Zhytnikov, Phys. Lett. B369 (1996) 215

[4] M. Reuter, hep-th/9605030 